\title{
COLLABORATIVE ADAPTIVE FILTERING IN THE COMPLEX DOMAIN
}

\author{
Beth Jelfs, Yili Xia and Danilo P. Mandic \\ Dept. of Electrical \& Electronic Engineering \\ Imperial College London \\ London, SW7 2BT UK
}

\author{
Scott C. Douglas
}

\author{
Dept. of Electrical Engineering \\ Southern Methodist University \\ Dallas, Texas 75275 USA
}

\begin{abstract}
A novel hybrid filter combining the complex least mean square (CLMS) and augmented CLMS (ACLMS) algorithms for complex domain adaptive filtering is introduced. The ACLMS has been shown to have improved performance in terms of prediction of non-circular complex data compared to that of the CLMS. By taking advantage of this along with the faster convergence of the CLMS, the hybrid filter is shown to give improved performance over both algorithms for both circular and non-circular data. Simulations on complex-valued synthetic and real world data support the effectiveness of this approach.
\end{abstract}

\section{INTRODUCTION}

Complex-valued signals can be either complex by design (communications) or by convenience of representation (radar, sonar). In fact representations in the complex domain provide a natural processing platform, for example processing real domain signals in $\mathbb{C}$ allows the inclusion of phase components, resulting in multidimensional solutions with benefits over real domain solutions [1]. One example of a real-valued signal which is best analysed in $\mathbb{C}$ is wind, where the fusion of the speed and direction creates a single complex-valued wind signal (see Fig. 7).

For adaptive filtering in the complex domain the complex least mean square (CLMS) algorithm [2] is a natural extension of the LMS algorithm [3] and benefits from the stability and robustness of the LMS while allowing simultaneous filtering of the real and imaginary parts of the complex-valued data. The CLMS is described by

$$
\begin{aligned}
e(k) & =d(k)-\mathbf{x}^{T}(k) \mathbf{w}(k), \\
\mathbf{w}(k+1) & =\mathbf{w}(k)+\mu e(k) \mathbf{x}^{*}(k),
\end{aligned}
$$

where $d(k)$ and $e(k)$ denote respectively the desired signal and output error at time instant $k$. For a filter of length, $N, \mathbf{x}(k)=[x(k-1), x(k-2), \ldots, x(k-N)]^{T}$ is the input signal vector, $\mathbf{w}(k)=\left[w_{1}(k), w_{2}(k), \ldots, w_{N}(k)\right]^{T}$ the filter weight coefficient vector and $\mu$ the learning rate. However, in general, the extensions of adaptive filters from $\mathbb{R}$ to $\mathbb{C}$ are non-trivial. For instance, in the design of nonlinear filters, it should be taken into account that the only continuously differentiable function in $\mathbb{C}$ is a constant (Liouville's theorem) and the differences between the statistics in $\mathbb{R}$ and $\mathbb{C}$ should be taken into account. Frequently though statistics in the complex domain are assumed to be straightforward extensions of those in the real domain. For example the covariance matrix of a zero mean complex vector, $\mathbf{z}$, is usually assumed to be an extension of the real covariance $E\left\{\mathbf{x x}^{T}\right\}$ replacing the transpose operator $(\cdot)^{T}$ with the Hermitian operator $(\cdot)^{H}$ to give $E\left\{\mathbf{x x}^{H}\right\}$ [4]. This however is only strictly true for circular complex data and does not hold true in many applications [5]. The use of so called augmented complex statistics takes into account not only the usually defined covariance matrix but also the pseudo-covariance matrix, whereby for any complex random vector $\mathbf{x} \in \mathbb{C}^{m}$ with $E\{\mathbf{x}\}=0$, the two covariance matrices are defined as

$$
\mathcal{C}_{\mathbf{x x}}=E\left\{\mathbf{x x}^{H}\right\}, \quad \mathcal{P}_{\mathbf{x x}}=E\left\{\mathbf{x x}^{T}\right\}
$$

where $\mathcal{C}_{\mathbf{x x}}$ and $\mathcal{P}_{\mathbf{x x}}$ are the covariance matrix and pseudocovariance matrix respectively [6]. For circular complex data the pseudo-covariance matrix $\mathcal{P}_{\mathbf{x x}}$ is zero, but for any noncircular data taking into account only the covariance matrix and not the pseudo-covariance matrix results in undermodelling.

By defining an augmented complex vector $\mathbf{x}_{a}$ as

$$
\mathbf{x}_{a}=\left[\begin{array}{l}
\mathbf{x} \\
\mathbf{x}^{*}
\end{array}\right]
$$

where $(\cdot)^{*}$ is the complex conjugate. Then the covariance matrix $\mathcal{C}_{\mathbf{x}_{\mathbf{a}} \mathbf{x}_{\mathbf{a}}}$ is given by

$$
\mathcal{C}_{\mathbf{x}_{\mathbf{a}} \mathbf{x}_{\mathbf{a}}}=\left[\begin{array}{cc}
\mathcal{C}_{\mathbf{x x}} & \mathcal{P}_{\mathbf{x x}} \\
\mathcal{P}_{\mathbf{x x}}^{*} & \mathcal{C}_{\mathbf{x x}}^{*}
\end{array}\right]
$$

and contains information from both the covariance and pseudo-covariance matrices of $\mathbf{x}$. To utilise the full second order statistical information available within the signal the recently introduced augmented CLMS (ACLMS) [7] uses the augmented complex vector as the input to an FIR filter. 
The ACLMS is given by

$$
\begin{aligned}
\mathbf{x}_{a}(k) & =\left[\mathbf{x}^{T}(k), \mathbf{x}^{H}(k)\right]^{T} \\
e(k) & =d(k)-\mathbf{x}_{a}^{T}(k) \mathbf{w}(k) \\
\mathbf{w}(k+1) & =\mathbf{w}(k)+\mu e(k) \mathbf{x}_{a}^{*}(k) .
\end{aligned}
$$

In this paper we propose a hybrid filter which following the approach from [8] combines the output of the CLMS with that of the ACLMS in a convex manner. Hybrid filters have been introduced to improve the performance of adaptive filters [9] and their convex combinations have been shown to always perform at least as well as the better of the two constituent subfilters and to offer improved stability as should one subfilter fail to converge the output of the hybrid filter follows that of the other subfilter [10]. By combining the ACLMS and CLMS our aim is to produce a filter with better overall characteristics for both circular and non-circular complex signals than either of the individual algorithms. This is supported with simulations on both synthetic and real world data.

\section{HYBRID FILTER STRUCTURE}

One of the keys to designing a hybrid filter is the method in which the subfilters are combined, for a hybrid filter containing only two subfilters one natural solution is to combine the outputs of the subfilters in a convex manner, where convexity is described by [11]

$$
\lambda x+(1-\lambda) y \text { where } \lambda \in[0,1] .
$$

If $x$ and $y$ are two points on a line, as shown in Fig. 1, their convex mixture (6) will lie on the same line between $x$ and $y$. The hybrid filter, shown in Figure 2, consists of two subfilters,

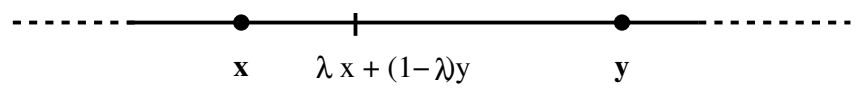

Fig. 1. Convex combination of two points $x$ and $y$.

each being adapted independently, with a convex combination of the two filters then taken as the output of the hybrid filter. The two subfilters within the hybrid filtering architecture operate in the prediction setting, sharing the common input vector $\mathbf{x}(k)=\left[x_{1}(k), \ldots, x_{N}(k)\right]^{T}$ for filters of length $N$, where to preserve its inherent characteristics each subfilter is updated by its own error $e_{1}(k)$ and $e_{2}(k)$, using a common desired signal $d(k)$. The convex combination of the subfilter outputs $y_{1}(k)$ and $y_{2}(k)$ forms the overall system output $y(k)$, given by

$$
y(k)=\lambda(k) y_{1}(k)+(1-\lambda(k)) y_{2}(k)
$$

where $\lambda(k)$ is the mixing parameter. The mixing parameter $\lambda(k)$ is made adaptive, and is updated by minimising the cost

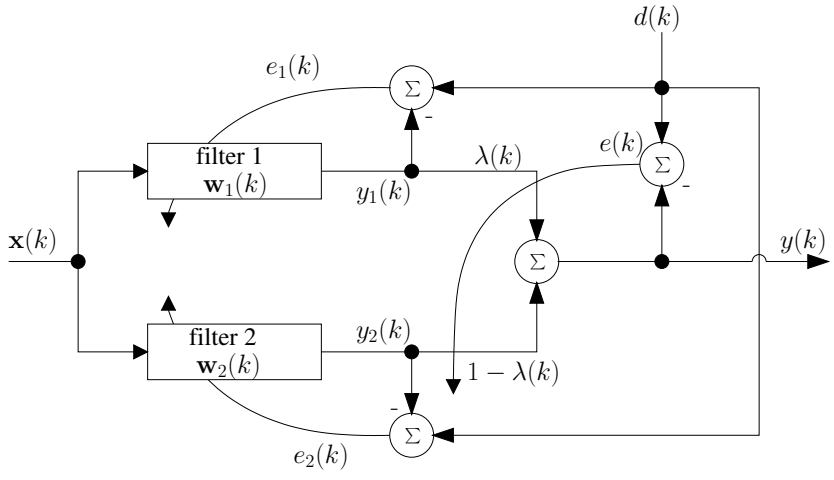

Fig. 2. Hybrid filter structure

function

$$
E(k)=\frac{1}{2}|e(k)|^{2}=\frac{1}{2}|d(k)-y(k)|^{2} .
$$

We can obtain the update for $\lambda(k)$ using a stochastic gradient based adaptation, such as the LMS, whereby

$$
\lambda(k+1)=\lambda(k)-\left.\mu_{\lambda} \nabla_{\lambda} E(k)\right|_{\lambda=\lambda(k)}
$$

and $\mu_{\lambda}$ is the step size. Note that since the input to the filters is complex, the error $e(k)$ is also complex, and therefore [12]

$$
\left.\nabla_{\lambda} E(k)\right|_{\lambda=\lambda(k)}=\left\{e(k) \frac{\partial e^{*}(k)}{\partial \lambda(k)}+e^{*}(k) \frac{\partial e(k)}{\partial \lambda(k)}\right\} .
$$

The two gradient terms from (10) can be evaluated as

$$
\begin{aligned}
\frac{\partial e(k)}{\partial \lambda(k)} & =\frac{\partial e_{r}(k)}{\partial \lambda(k)}+\jmath \frac{\partial e_{i}(k)}{\partial \lambda(k)}, \\
\frac{\partial e^{*}(k)}{\partial \lambda(k)} & =\frac{\partial e_{r}(k)}{\partial \lambda(k)}-\jmath \frac{\partial e_{i}(k)}{\partial \lambda(k)},
\end{aligned}
$$

where $(\cdot)_{r}$ and $(\cdot)_{i}$ denote respectively the real and imaginary part of a complex number. Rewriting (7) in terms of its real and imaginary part and substituting into (8) yields

$$
\begin{gathered}
\frac{\partial e(k)}{\partial \lambda(k)}=y_{1}(k)-y_{2}(k), \\
\frac{\partial e^{*}(k)}{\partial \lambda(k)}=\left(y_{1}(k)-y_{2}(k)\right)^{*} .
\end{gathered}
$$

Finally, the gradient (10) becomes

$$
\left.\nabla_{\lambda} E(k)\right|_{\lambda=\lambda(k)}=\Re\left\{e(k)\left(y_{1}(k)-y_{2}(k)\right)^{*}\right\},
$$

where $\Re(\cdot)$ denotes the real part of a complex number. This yields the mixing parameter update as

$$
\lambda(k+1)=\lambda(k)+\mu_{\lambda} \Re\left\{e(k)\left(y_{1}(k)-y_{2}(k)\right)^{*}\right\} .
$$

To ensure the hybrid filter remains a convex function a hard limit is put on the adaptation of $\lambda$ resctricting the values to the range $[0,1]$. 


\section{SIMULATIONS}

For all simulations conducted the hybrid filter described in the previous section was used with filter 1 being trained by the ACLMS and filter 2 being trained by the CLMS. Initial simulations were performed to illustrate the behaviour of the subfilters of the hybrid filter for both circular and non-circular data, Fig. 3 and highlight the advantages of using the hybrid filter over either of the constituent subfilters. The circular signal used was a stable AR(4) process given by

$$
\begin{aligned}
x(k) & =1.79 x(k-1)-1.85 x(k-2)+1.27 x(k-3) \\
& -0.41 x(k-4)+n(k),
\end{aligned}
$$

where $n(k)$ is a random uniform circular variable given by

$$
n(k)=\rho(k) \cos (\theta(k))+j \rho(k) \sin (\theta(k))
$$

and $\rho$ and $\theta$ are random variables uniformly distributed on the unit interval and $[0,2 \pi]$ respectively. The non-circular data used was the Ikeda map, a well known benchmark signal in chaos theory [13]

$$
\begin{aligned}
& x(k+1)=1+u[x(k) \cos t(k)-y(k) \sin t(k)] \\
& y(k+1)=u[x(k) \sin t(k)+y(k) \cos t(k)]
\end{aligned}
$$

where $u$ is a parameter and

$$
t(k)=0.4-\frac{6}{1+x^{2}(k)+y^{2}(k)} .
$$

All simulations were averaged over a set of 1000 independent trials for filters of length $N=100$. From Fig. 4 it can be seen that both the CLMS and ACLMS perform well on the circular data, with the CLMS converging slightly faster than the ACLMS. This is to be expected as the ACLMS is in effect twice the length of the CLMS and therefore takes longer to converge. The key point to note here is that in this instance the hybrid filter outperforms both of its constituent subfilters converging slightly faster and with an improved steady state error.

For the non-circular data shown in Fig. 5 the CLMS again converges faster than the ACLMS but in this instance does not perform as well in the steady state as the ACLMS. Again the hybrid filter outperforms both subfilters by following first the CLMS and then the slower ACLMS when it surpasses the CLMS to give a better combined output.

The evolution of the mixing parameter $\lambda$ for both data sets is illustrated in Fig. 6. In this case one represents the output of the hybrid filter is purely that of the ACLMS and zero indicates it is purely the CLMS. For both sets of simulations the initial value of the mixing parameter was set to be 0.5 and it can clearly be seen that for the circular data the value of $\lambda$ tends slightly towards that of the CLMS but there is no distinct advantage gained from either algorithm. In the case of the non-circular data the value of $\lambda$ initially moves quickly

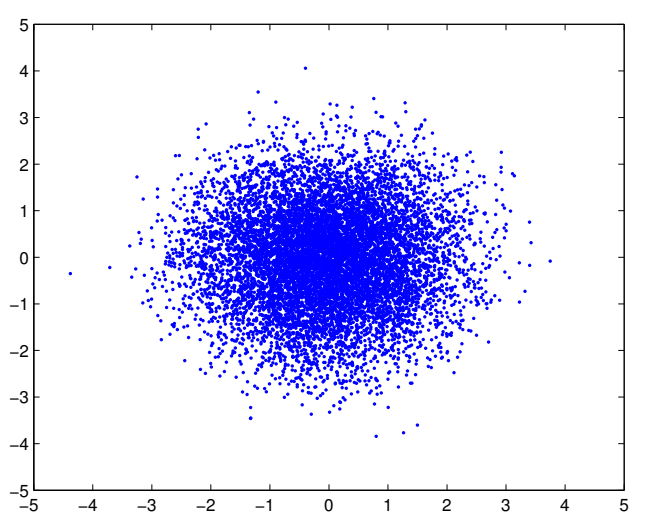

(a) A circular AR(4) process (17)

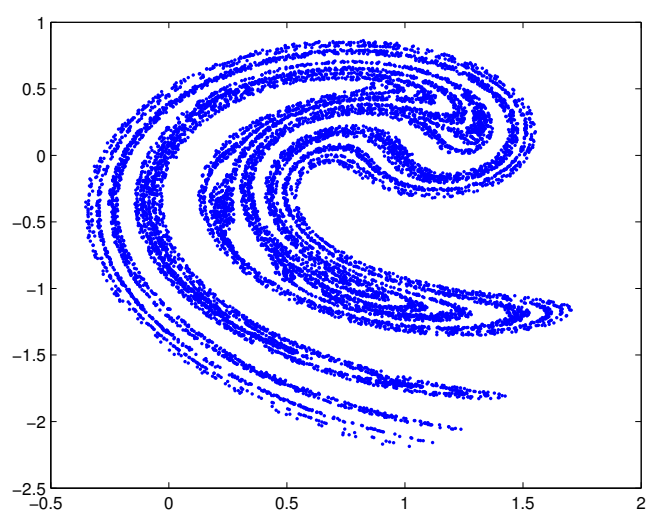

(b) Non-circular Ikeda map (19)

Fig. 3. Distributions of the circular and non-circular benchmark signals

towards that of the CLMS before it eventually settles towards that of the ACLMS. In both cases the value of the $\lambda$ is as expected and supports the observed outputs of the hybrid filter.

\subsection{Prediction of Wind Data}

A set of wind data was analysed to illustrate the performance of the hybrid filter for real world applications. Wind is usually measured as a bivariate process of its speed and direction [14] or even just the speed is taken into account (despite clear interdependence between the components). Figure 7 shows wind represented as a vector of speed and direction in the northeast coordinate system. It is clear that the wind vector $\mathbf{v}$ can be represented in the complex domain as

$$
\mathbf{V}=\mathbf{v} \cdot e^{j \theta}
$$

where $\mathbf{v}$ is the speed and $\theta$ the direction modelled as a single complex value. 


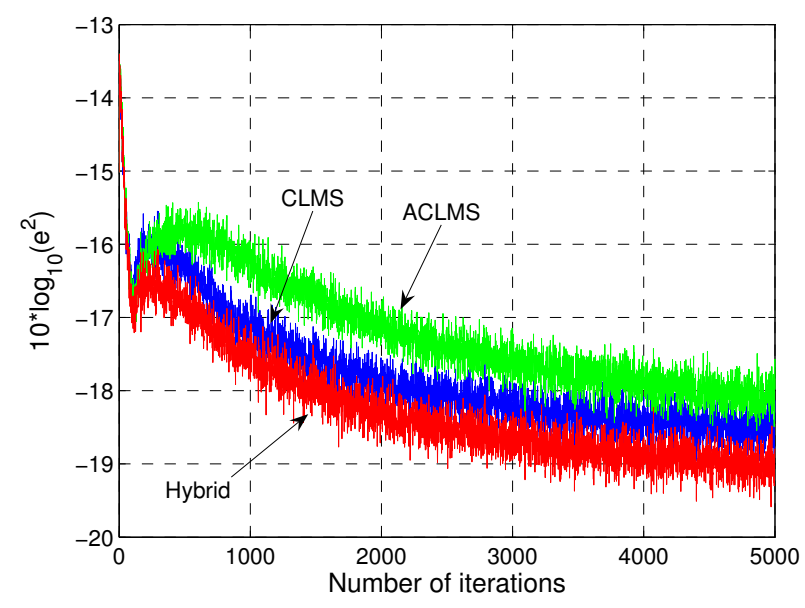

Fig. 4. Convergence curves of the hybrid filter and constituent subfilters for a circular AR(4) process (17)

\begin{tabular}{c|cccc} 
& AR(4) & Ikeda & 'Calm' wind & 'High' wind \\
\hline CLMS & 5.25 & 0.65 & 7.03 & 3.26 \\
ACLMS & 4.73 & $\mathbf{3 . 7 7}$ & 6.87 & 4.35 \\
Hybrid & $\mathbf{5 . 6 6}$ & 3.73 & $\mathbf{7 . 3 3}$ & $\mathbf{4 . 4 8}$
\end{tabular}

Table 1. Prediction gain for the CLMS, ACLMS and hybrid filters for wind data and circular and non-circular synthetic data

The wind data used was measured over a 24 hour period sampled at $50 \mathrm{~Hz}$ in an urban environment. The wind speed readings were taken in the north-south $\left(\mathbf{V}_{N}\right)$ and east-west $\left(\mathbf{V}_{E}\right)$ directions where

$$
\mathbf{v}=\sqrt{\mathbf{V}_{E}^{2}+\mathbf{V}_{N}^{2}}, \quad \theta=\arctan \left(\frac{\mathbf{V}_{N}}{\mathbf{V}_{E}}\right)
$$

were used to give the complex signal (21). To reduce the effects of high frequency noise a moving average filter with window length 10s was applied to the data before resampling at $1 \mathrm{~Hz}$. The magnitude of the wind signal is shown in Fig. 8, to best assess the performance of the hybrid filter two periods of approximately 90 mins were assessed one from the 'calm' period between 04:00-05:30 and one from the 'high' wind state between 16:00-17:30.

The prediction gains $R_{p}=10 \log _{10} \frac{\sigma_{y}^{2}}{\sigma_{e}^{2}}$ of the subfilters and hybrid filters in the steady state were used as a quantitative measure of performance. Table 1 lists the prediction gains for the calm and high wind periods along with those for the circular AR(4) signal and non-circular Ikeda map. For the wind data it can be seen from Table 1 that in the calm wind state the CLMS performs better than the ACLMS in the steady state but for the high wind state where there are large variations in the signal dynamics the ACLMS performs better than the CLMS. It should also be noted that more significantly in all but one case the hybrid filter outperforms both of the sub-

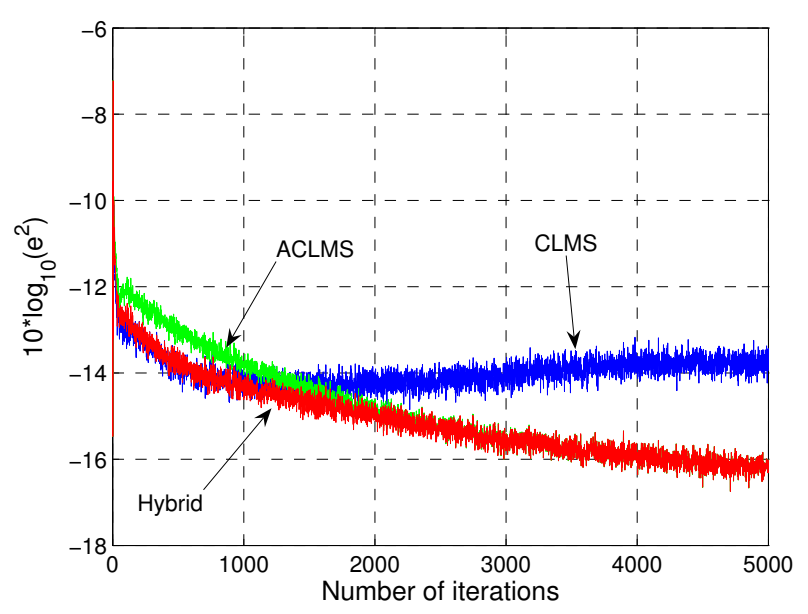

Fig. 5. Convergence curves of the hybrid filter and constituent subfilters for a non-circular Ikeda map (19)

filters regardless of which subfilter is responding best to the current input. In the case of the non-circular Ikeda map the hybrid filter only fractionally under performs the ACLMS in terms of steady state prediction gain and as illustrated in Fig. 5 has a faster convergence curve than the ACLMS.

\section{CONCLUSIONS}

A hybrid filter consisting of a convex combination of the CLMS and ACLMS algorithms has been introduced. The hybrid filter takes advantage of the faster convergence speeds of the CLMS and the improved performance in the steady state for non-circular data of the ACLMS. It has been shown that the hybrid filter can outperform both of the subfilters for synthetic circular and non-circular data. A real world wind signal has been used to demonstrate that for signals where the nature may be changing the hybrid filter will perform consistently well regardless of which subfilter has the better performance for the current input signal.

\section{ACKNOWLEDGEMENT}

We would like to thank Prof. Kazayuki Aihara and Dr Yoshito Hirata from the Institute of Industrial Science, University of Tokyo, Japan for providing the wind data sets.

\section{REFERENCES}

[1] D.P. Mandic, S. Javidi, G. Souretis, and S.L. Goh, "Why a complex valued solution for a real domain problem," in Proceedings IEEE International Workshop on Machine Learning for Signal Processing, 2007, pp. 384389. 


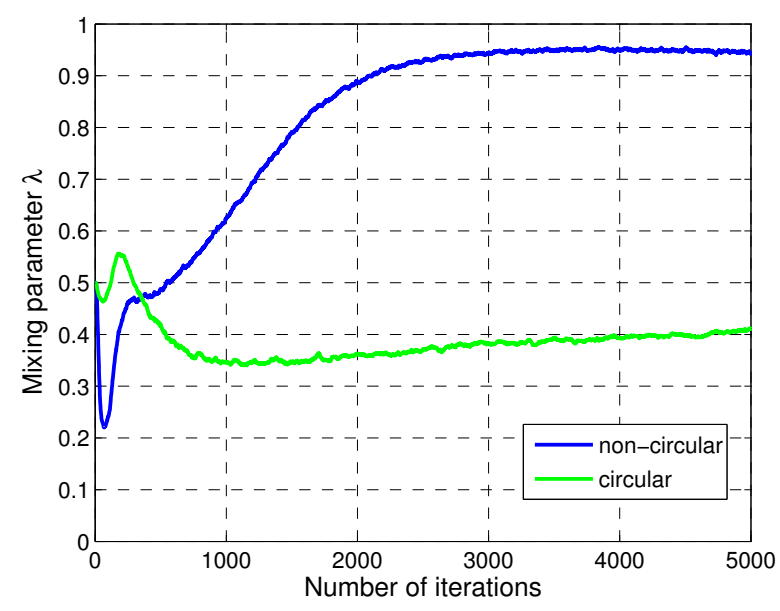

Fig. 6. Behaviour of the mixing parameter $\lambda$ for circular and non-circular data

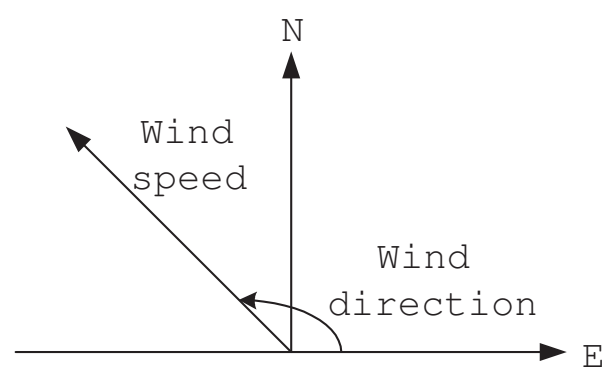

Fig. 7. Wind recordings as a complex [speed,direction] vector representation

[2] B. Widrow, J. McCool, and M. Ball, "The complex LMS algorithm," Proceedings of the IEEE, vol. 63, no. 4, pp. 719-720, 1975.

[3] B. Widrow and S.D. Stearns, Adaptive Signal Processing, Prentice-Hall, 1985.

[4] P.J. Schreier and L.L. Scharf, "Second-order analysis of improper complex random vectors and processes," IEEE Transactions on Signal Processing, vol. 51, no. 3, pp. 714-725, 2003.

[5] B. Picinbono, "On circularity," IEEE Transactions on Signal Processing, vol. 42, no. 12, pp. 3473-3482, 1994.

[6] F.D. Neeser and J.L. Massey, "Proper complex random processes with applications to information theory," IEEE Transaction on Information Theory, vol. 39, no. 4, pp. 1293-1302, 1993.

[7] S. Javidi, M. Pedzisz, S.L. Goh, and D.P. Mandic, "The augmented complex least mean square algorithm with

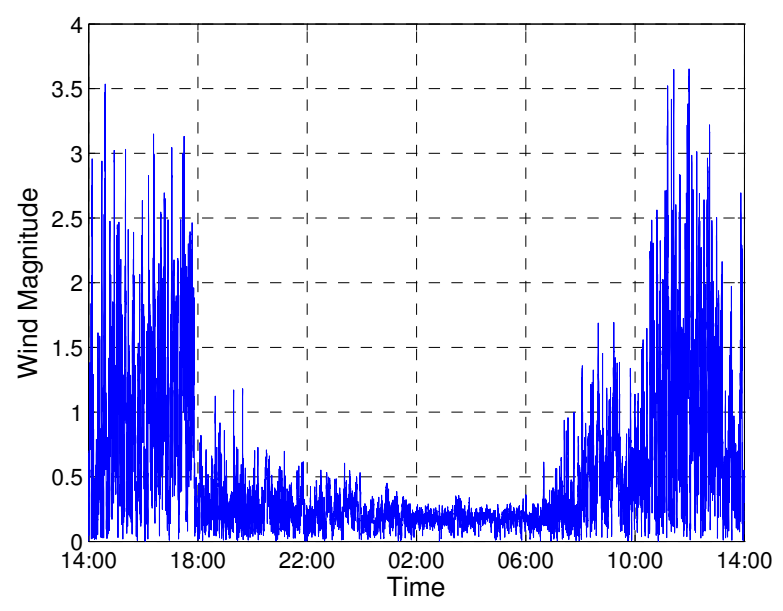

Fig. 8. Wind magnitude over a 24 hour period in an urban environment

application to adaptive prediction problems," in Proceedings of the IAPR Workshop on Cognitive Information Processing, 2008.

[8] D. Mandic, P. Vayanos, C. Boukis, B. Jelfs, S.L. Goh, T. Gautama, and T. Rutkowski, "Collaborative adaptive learning using hybrid filters," in Proceedings IEEE International Conference on Acoustics, Speech and Signal Processing, ICASSP 2007, 2007, vol. 3, pp. 921-924.

[9] S.S. Kozat and A.C. Singer, "Multi-stage adaptive signal processing algorithms," in Proceedings of the IEEE Sensor Array and Multichannel Signal Processing Workshop, 2000, pp. 380-384.

[10] J. Arenas-Garcia, A.R. Figueiras-Vidal, and A.H. Sayed, "Mean-square performance of a convex combination of two adaptive filters," IEEE Transactions on Signal Processing, vol. 54, no. 3, pp. 1078-1090, 2006.

[11] A. Cichocki and R. Unbehauen, Neural Networks for Optimisation and Signal Processing, Wiley, 1993.

[12] D.P. Mandic, P. Vayanos, S. Javidi, B. Jelfs, and K. Aihara, "Online tracking of the degree of nonlinearity within complex signals," in Proceedings IEEE International Conference on Acoustics, Speech and Signal Processing, ICASSP 2008, 2008, pp. 2061-2065.

[13] K. Aihara, Ed., Applied Chaos and Applicable Chaos, Tokyo: Science-Sha, 1994.

[14] M.C. Alexiadis, P.S. Dokopoulos, H.S. Sahsamanoglou, and I.M. Manousadiris, "Short-term forecasting of wind speed and related electrical power," Solar Energy, vol. 63, no. 1, pp. 61-68, 1998. 